\title{
On the Occurrence of Naked Singularities in General Relativity. II $^{\star}$
}

\author{
H. Müller zum Hagen and P. Yodzis \\ I. Institut für Theoretische Physik, Universität Hamburg, Hamburg \\ Federal Republic of Germany \\ H.-J. Seifert \\ Hochschule der Bundeswehr Hamburg, Fachbereich Maschinenbau, Hamburg, \\ Federal Republic of Germany
}

Received October 1, 1973

\begin{abstract}
It is shown that naked "shell crossing" singularities can occur in the gravitational collapse of a spherically symmetric ball of perfect fluid for a large family of equations of state in which the pressure has an (arbitrarily large) upper bound, and, moreover, that this behaviour is stable with respect to spherically symmetric perturbations of the initial data, as well as with respect to perturbations of the equation of state.
\end{abstract}

\section{Introduction}

In a previous paper [1], we displayed explicit solutions of Einstein's field equations for the gravitational collapse of an isolated, spherically symmetric object, in which regular initial data evolve into naked singularities - contrary to the widely accepted [2] conjecture that, according to general relativity, collapse inevitably leads to black holes which hide the singularities. In one family of these solutions (inhomogeneous dust clouds), the singularities are associated with "shell crossing", or caustics in the congruence of matter flow lines. One might be inclined to dismiss these solutions by asserting that i) such caustics become quite innocuous if the pressure is nonzero, ii) these singularities are in any case unstable.

We shall, however, show here that naked "shell crossing" singularities can occur in the collapse of a spherically symmetric ball of perfect fluid for a large family of equations of state in which the pressure has an (arbitrarily large) upper bound, and, moreover, that this behaviour is stable with respect to (spherically symmetric) initial conditions and equation of state.

The restriction of bounded pressure is not necessarily so unphysical as it may seem. Our upper bound can be arbitrarily large, say $10^{114} \mathrm{erg} / \mathrm{cm}^{3}$

* Work supported by the Deutsche Forschungsgemeinschaft. 
(corresponding to the "Planck length" for radius of curvature). It seems quite likely that long before such pressures could be attained, the concept of classical spacetime (and with it, classical general relativity) would anyway become physical nonsense.

We restrict, as usual, our attention to spacetimes which contain only a single object ${ }^{1}$. We say that this object is undergoing gravitational collapse if 1) the spacetime contains a regular ("initial") spacelike hypersurface on which the energy-momentum tensor has connected compact support and all physical quantities are well behaved, 2) there exists a nonextendible causal curve within the matter which has finite affine length. We say that the singularity is naked if there exists a causal curve with one end on $\mathscr{I}^{+}$and the other end "on the singularity".

It should be noted that we are treating dynamical solutions which evolve from regular initial data into singularities. There are many well known static solutions which have naked singularities, but their physical significance is far from clear, and they are excluded from the present discussion by condition 1) of our definition of gravitational collapse.

Our procedure here is the following. We pose a Cauchy problem, but instead of giving regular initial data, we specify final data on a spacelike hypersurface such that the energy density becomes infinite on this hypersurface. We show that with this final data our Cauchy problem has a regular solution into the past. Then taking as initial surface some spacelike hypersurface in the past of our final surface, one sees that there exist regular initial data which evolve (now forward in time) into a naked singularity.

In Section II we write down Einstein's field equations and state our Cauchy problem. In Section III we discuss the specification of appropriate final data, including a condition for the presence of naked singularities. In Section IV we show that our Cauchy problem is well posed and prove the existence and uniqueness of solutions into the past, and the stability of the occurrence of the corresponding naked singularities. Section V contains a concise statement of our results, along with a few remarks; the appendices treat technical details.

\section{Statement of the Cauchy Problem}

We consider the isentropic collapse of a sphere of perfect fluid, assuming that the specific entropy is uniform throughout the sphere. Then the equation of state can be specified by giving, say, pressure $p$ as a function of energy density $\varepsilon$. If $u$ is the specific internal energy

1 In physical terms, this means that the collapsing object is assumed sufficiently far away from everything else in the universe so that everything else can be neglected. 
(including rest mass) and $v$ the specific volume ${ }^{2}$ of a unit quantity of matter, then $\varepsilon=u / v$.

Because of our thermodynamical assumptions one can, if one prefers, write the pressure as a function of $v[3]$; this form is more convenient for us. Set

$$
v_{1}:=\sup \{v \mid p(v)>0\} ;
$$

we neglect any atmosphere surrounding the object by assuming that $v_{1}$ is finite; then $p\left(v_{1}\right)=0$.

We shall use a slightly modified version of the equations derived by Misner and Sharp $[3,4]$ for spherically symmetric perfect fluids. We write the spacetime metric in the form

$$
d s^{2}=e^{\lambda(r, t)} d r^{2}+R^{2}(r, t) d \Omega^{2}-e^{2 \varphi(r, t)} d t^{2}
$$

where $r$ is a comoving radial coordinate.

The number $A$ of unit quantities of matter contained in a sphere of coordinate radius $r$,

$$
A(r):=\int_{0}^{r} 4 \pi R^{2} e^{\lambda / 2} v^{-1} d r,
$$

is independent of $t$, and we shall choose our comoving radial coordinate ${ }^{3}$ as $r:=(3 A / 4 \pi)^{1 / 3}$. We define, with Misner and Sharp, the quantities

$$
\begin{aligned}
& U(r, t):=D_{t} R=e^{-\varphi} \dot{R} ; \quad \Gamma(r, t):=D_{r} R=e^{-\lambda / 2} R^{\prime} ; \\
& m(r, t):=\int_{0}^{r} 4 \pi \varepsilon R^{2} R^{\prime} d r
\end{aligned}
$$

where $^{\prime}:=\frac{\partial}{\partial r}$ and ${ }^{\prime}:=\frac{\partial}{\partial t}$. We shall take as our basic functions $U, v, R, \Gamma$. The metric coefficients can be written $[3,4]$ as (recall that $u$ and $p$ are functions of $v$ )

$$
e^{\lambda / 2}=\frac{r^{2} v}{R^{2}} ; \quad e^{\varphi}=\frac{1}{u+p v},
$$

so clearly our four basic functions fully determine a solution. Einstein's field equations are in fact equivalent [4] to the constraint equations

$$
\Gamma=\frac{R^{2} R^{\prime}}{r^{2} v} ; \quad \Gamma^{2}-U^{2}-1+\frac{2 m}{R}=0
$$

along with the system of evolution equations

$$
\dot{f}^{i}+A_{k}^{i}\left(r, t, f^{j}\right) f^{k \prime}+B^{i}\left(r, t, f^{j}\right)=0,
$$

${ }^{2}$ Note that $v=n^{-1}$, where $n$ is the number density. For our purposes, $v$ is a vastly preferable quantity to $n$, since $v$ is finite (in fact, zero) at the singularity.

${ }^{3}$ This choice for $r$ has two nice properties: it is proportional, in first order, to the radial distance to the center; and our system is hyperbolic with this choice [see Section IV]. 
provided that $\Gamma>0$ everywhere. Here we have defined

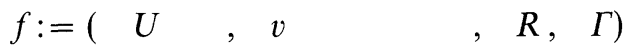

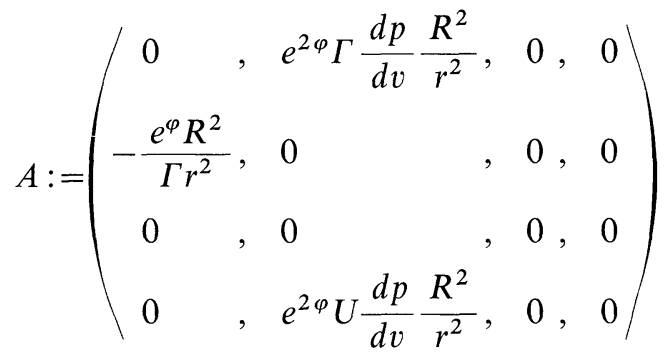

$$
\begin{aligned}
& B:=\left(e^{\varphi}\left(4 \pi p R+\frac{1+U^{2}-\Gamma^{2}}{2 R}\right),-e^{\varphi} \frac{2 U v}{R},-e^{\varphi} U, 0\right) .
\end{aligned}
$$

If the constraint Eqs. (6) hold at one time, then the evolution Eqs. (7) imply that they hold at all times.

Our set of basic functions is redundant; in fact two basic functions suffice to determine the solution $[3,4]$. But there is no harm in this redundancy, and it has the virtue that the evolution Eqs. (7) for our set of basic functions are quasilinear.

We consider now the Cauchy problem for these equations. Let $r \in I:=[0, b]$ be the region occupied by matter. The appropriate Cauchy data for this system of equations are "mixed": in addition to giving the four functions $f^{i}\left(I, t_{0}\right)$ at some time $t_{0}$, one must specify the boundary conditions $U(0, t)=0$ and $v(b, t)=v_{1}$. The first of these boundary conditions ensures that the line $r=0$ is a regular center of spherical symmetry, and the second ensures that the pressure is zero [recall Eq. (1)] at the junction with the vacuum $r=b$, which is the necessary and sufficient condition for this interior solution to match to a vacuum exterior [3].

We shall show in Section IV that this really is a properly posed Cauchy problem. For the moment, we note that in order for the solution to be $C^{2}$, we must impose on the initial data the consistency conditions $\dot{U}\left(0, t_{0}\right)=0, \ddot{U}\left(0, t_{0}\right)=0, \dot{v}\left(b, t_{0}\right)=0, \ddot{v}\left(b, t_{0}\right)=0[5 ; \mathrm{p} .472]$.

\section{Final Data}

Let $t=t_{s}$ be the final hypersurface. We want to specify the four functions $f^{i}\left(I, t_{s}\right)$ in such a way that our Cauchy problem has a regular solution into the past, but the corresponding spacetime has a naked singularity ${ }^{4}$. So we first need a condition on our final data which ensures the presence of a naked singularity; this is provided by the following

\footnotetext{
${ }^{4}$ For details about some possible causal structures of such spacetimes see Ref. [1].
} 
Lemma. Suppose $v\left(r_{s}, t_{s}\right)=0, r_{s} \in I ; R\left(r, t_{s}\right)$ is monotonically increasing for $r \in\left[r_{s}, b\right]$; and $R\left(r_{s}, t_{s}\right)>2 m\left(b, t_{s}\right)$. Then if a solution to the Cauchy problem exists, it has (when maximally extended) a naked singularity.

Proof. The mass of the Schwarzschild exterior to which this interior solution matches is $m(b, t)$ [3]. But the surface area of $t=$ constant sections of the horizon cannot increase into the past [6;p.318], so $R \leqq 2 m\left(b, t_{s}\right)$ everywhere on the horizon when the horizon is extended into the interior. On the other hand, it follows from our hypotheses that $R\left(r, t_{s}\right)>2 m\left(b, t_{s}\right)$ for all $r \in\left[r_{s}, b\right]$. The point $\left(b, t_{s}\right)$ is certainly outside the horizon, and since $R \leqq 2 m\left(b, t_{s}\right)$ everywhere on the horizon, no point of the horizon can lie in the past of the set $\left\{r \in\left[r_{s}, b\right] ; t=t_{s}\right\}$. Therefore either the singularity at $\left(r_{s}, t_{s}\right)$ can communicate with $\mathscr{I}^{+}$, or at least the horizon encounters some other naked singularity.

We can now list all the conditions to be fulfilled by our final data. There are five sets of conditions:

a) Constraint equations: $\Gamma=\frac{R^{2} R^{\prime}}{r^{2} v} ; \Gamma^{2}-U^{2}-1+\frac{2 m}{R}=0$;

b) shell crossing singularity at $\left(r_{s}, t_{s}\right)$, with regularity to the past: $v\left(r_{s}, t_{s}\right)=0 ; v\left(r, t_{s}\right) \in\left(0, v_{1}\right)$ for $r \in I, r_{s} \neq r \neq b ; R^{\prime}\left(r_{s}, t_{s}\right)=0 ; R^{\prime}\left(r, t_{s}\right)>0$ for $r \in I, r_{s} \neq r ; \dot{v}\left(r_{s}, t_{s}\right)<0$;

c) boundary and consistency conditions: $R\left(0, t_{s}\right)^{\bullet}=0 ; U\left(0, t_{s}\right)=0$; $\dot{U}\left(0, t_{s}\right)=0 ; \ddot{U}\left(0, t_{s}\right)=0 ; v\left(b, t_{s}\right)=v_{1} ; \dot{v}\left(b, t_{s}\right)=0 ; \ddot{v}\left(b, t_{s}\right)=0 ;$

d) nakedness conditions: $\left.R\right|_{t=t_{s}}$ monotone, $R\left(r_{s}, t_{s}\right)>2 \mathrm{~m}\left(b, t_{s}\right)$;

e) smoothness conditions: $U, v, R, \Gamma$ are $C^{2}$ on $\left\{t=t_{s}\right\}, \Gamma>0$.

We must show that these conditions are compatible. We shall proceed by expressing all our data in terms of two functions $m\left(I, t_{s}\right)$ and $R\left(I, t_{s}\right)$. First, we have

$$
\varepsilon=\frac{m^{\prime}}{4 \pi R^{2} R^{\prime}} .
$$

But since $v$ is determined as a function of $\varepsilon$ by the equation of state, this equation fixes $v$. Then the first constraint equation fixes $\Gamma$, after which the second constraint equation fixes $U$ to within its sign. This sign we are free to choose; we shall fix it in the following paragraph. With this procedure, conditions a) are of course automatically satisfied.

We now show how to choose $R$ and $m$ in such a way that the remaining conditions are also fulfilled. First, choose $r_{s}, b$, and a monotonically increasing $C^{3}$ function $m(r)$, with $m(0)=0, m^{\prime}(r)=0$ at and only at $r=0$, and

where

$$
\begin{gathered}
m(b)^{2}<\frac{3}{32 \pi \varepsilon\left(v_{1}\right)} \cdot \frac{m\left(r_{s}\right)}{m(b)} . \\
m^{\prime} \geqq 4 \pi r^{2} u_{0}
\end{gathered}
$$

$$
u_{0}=u\left(v_{1}\right)+\int_{0}^{v_{1}} p d v
$$


If (as we shall assume later) $p(v)$ has an upper bound $p_{m}$, then $u_{0}<u\left(v_{1}\right)$ $+p_{m} v_{1}$. Then choose a monotonically increasing $C^{3}$ function $R(r)$, with $R(0)=0, R\left(r_{s}\right)>2 m(b), R^{\prime}(r)=0$ at and only at $r=r_{s}$, and

$$
\begin{gathered}
m^{\prime \prime}\left(r_{s}\right) r_{s} R\left(r_{s}\right)-2 m^{\prime}\left(r_{s}\right) R\left(r_{s}\right)+\left.16 \pi^{2} r_{s}^{5} u\right|_{v=0} ^{2} \neq 0, \\
R^{2} R^{\prime} \leqq m^{\prime} / 4 \pi \varepsilon\left(v_{1}\right), \quad \text { with equality at, and only at } r=b .
\end{gathered}
$$

Conditions d) are obviously satisfied. If, having obtained $U^{2}$ by means of the constraint equations, the sign of $U$ is chosen such that $U^{\prime}\left(r_{s}\right)<0$ (which implies that $\dot{v}\left(r_{s}\right)<0$ ), conditions b) will also be satisfied [Eq. (11c) ensures that $\left.U^{\prime}\left(r_{s}\right) \neq 0\right]$. Equation $(11 \mathrm{~d})$ is just the condition $v=v_{1}$ at the boundary $r=b$, with $v<v_{1}$ for $r \in[0, b)$.

Equation (11a) is a consistency relation between (11d) and the nakedness conditions. Unfortunately, it does not give any significant restrictions on the occurence of naked shell crossing singularities. First, it could surely be weakened (since the nakedness conditions are sufficient, but certainly not necessary ones), and in any case it is already very weak from a physical point of view. For instance, if we take $\varepsilon\left(v_{1}\right)$ equal to the density of water and $m\left(r_{s}\right) / m(b)=10^{-2}$, then (11a) only implies that $m(b)<4 \cdot 10^{7}$ solar masses ${ }^{5}$.

Equation (11b) implies that $\Gamma>1$, which ensures that $U$, obtained from the second constraint equation, is real. A sufficient condition for the compatibility of (11a) and (11b) is

$$
b^{3}-r_{s}^{3} \leqq \frac{3}{4 \pi u_{0}}\left[m(b)-32 \pi \varepsilon\left(v_{1}\right) m(b)^{3}\right],
$$

which can certainly be fulfilled with $r_{s}<b$.

Finally, we note that the conditions c) and e) only add (via the evolution equations) restrictions on the first three derivatives of $R$ and $m$, evaluated at $r=0$ and $r=b$, which obviously can be fulfilled. It makes sense to have restrictions on the third derivatives of $R(r)$ and $m(r)$ because if all four of our basic functions $\left.f\right|_{t=t_{s}}(r)$ are $C^{2}$, this implies that $\left.R\right|_{t=t_{s}}(r)$ and $\left.m\right|_{t=t_{s}}(r)$ are $C^{3}$.

\section{Existence, Uniqueness, and Stability}

We return now to a consideration of the evolution Eqs. (7)-(10). For a quasilinear system (in two variables) of the form (7), with a set of initial data $\left.f^{i}\right|_{t=t_{0}}(r)=\psi^{i}(r)$, Courant and Hilbert [5; p. $461 \mathrm{ff}$. $]$ show by

\footnotetext{
${ }^{5}$ Given the minimum energy density $\varepsilon\left(v_{1}\right)$, a necessary condition for the occurrence of any naked singularity is ( $11 \mathrm{a})$ with $r_{s}=b$. But this inequality is precisely equivalent to the demand that the surface of the object is not a trapped surface, which is surely a natural requirement.
} 
an iteration procedure that there exists a unique solution possessing Lipschitz continuous first derivatives in a suitable neighbourhood $\left\{t \in\left[t_{0}-h, t_{0}\right]\right\}$ if the following conditions hold:

a) the system is hyperbolic with respect to the given initial data;

b) the initial data $\psi$, the coefficients $A, B$, and the left eigenvectors $l^{k}$ and the corresponding eigenvalues $\lambda^{k}$ of the matrix $A$ possess Lipschitz continuous first derivatives with respect to $r, t$, and $f$. It is not hard to see that if the condition of Lipschitz continuity of first derivatives in $b$ ) is replaced by the demand that these functions should all be $C^{2}$, then the solution will be $C^{2}$ in a suitable neighbourhood; let us call this condition $\left.b^{\prime}\right)$.

Under condition $b^{\prime}$ ), the spacetime metric is, as one wants, $C^{2}$ everywhere in the regular region. However, the fact that the solution to our system of equations continues to be $C^{2}$ also at the singularity does not mean that the spacetime is $C^{2}$ at the singularity (this would contradict the circumstance, already built into our final data, that the Ricci scalar is infinite at the singularity). There is no inconsistency here: One need only note that no comoving radial coordinate can be admissible at a shell crossing singularity.

The system is hyperbolic with respect to the data $\psi$ if and only if the matrix $A$ has four linearly independent left eigenvectors $l^{k}$,

$$
l_{i}^{k} A_{j}^{i}=\lambda^{k} l_{j}^{k},
$$

when the initial data are inserted in place of $f$ everywhere in $A[5 ;$ pp. 425 , 476]. In our case, the left eigenvectors can be chosen as

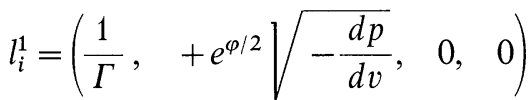

$$
\begin{aligned}
& l_{i}^{2}=\left(\begin{array}{ll}
\frac{1}{\Gamma}, & -e^{\varphi / 2} \sqrt{-\frac{d p}{d v}}, 0,0
\end{array}\right) \\
& l_{i}^{3}=\left(\begin{array}{lll}
0 & , 0
\end{array}\right. \\
& l_{i}^{4}=\left(\begin{array}{lll}
-U, & 0 \quad
\end{array}\right.
\end{aligned}
$$

These are linearly independent with respect to our final data if $d p / d v$ is strictly negative.

The smoothness condition $\mathrm{b}^{\prime}$ ) holds everywhere except at $r=0$ if the equation of state is such that $p(v)$ is of class $C^{3}$ in the closed interval $\left[0, v_{1}\right]$; this implies in particular that the pressure $p$ must be bounded, and that the speed of sound $\sqrt{d p / d \varepsilon}$ must tend to zero as $\varepsilon$ tends to infinity, in such a way that $\varepsilon^{2} d p / d \varepsilon$ is bounded. Within the set of bounded equations of state, this does not seem to be a very strong restriction: for instance, it holds for any equation of state for which $p(\varepsilon)$ is an inverse 
power series. Moreover, our restrictions on the equation of state are compatible with the physical requirement $d p / d \varepsilon<1$.

As for the failure of the smoothness condition at $r=0$, we shall show in Appendix I that the existence proof can be modified so as to admit this violation of the smoothness condition. Obviously, this is a mere technicality; the fact that it can be done just means that one can treat perfect fluids with regular centers.

For our mixed Cauchy problem, we must show in addition that we have specified our boundary conditions properly. Let $g^{k}$ denote the canonical functions for our system:

$$
g^{k}:=l_{i}^{k} f^{i} \text {. }
$$

In every iteration step, the functions $g^{k}$ propagate along the $k^{\text {th }}$ characteristic, that is, along the curves for which $d r / d t=\lambda^{k}$. For our system,

$$
\begin{aligned}
& \left(g^{1} ; \lambda^{1}\right)=\left(\frac{U}{\Gamma}+e^{\varphi / 2} \sqrt{-\frac{d p}{d v}} v ; \quad+e^{\varphi} e^{-\lambda / 2} \sqrt{\frac{d p}{d \varepsilon}}\right) \\
& \left(g^{2} ; \lambda^{2}\right)=\left(\frac{U}{\Gamma}-e^{\varphi / 2} \sqrt{-\frac{d p}{d v}} v ;-e^{\varphi} e^{-\lambda / 2} \sqrt{\frac{d p}{d \varepsilon}}\right) \\
& \left(g^{3} ; \lambda^{3}\right)=(R \\
& \left(g^{4} ; \lambda^{4}\right)=\left(-U^{2}+\Gamma^{2}\right. \\
& 0 \\
& 0
\end{aligned}
$$

It is seen that only the characteristic numbered 2 emanates from the "corner" $\left(0, t_{s}\right)$ into the region $\left\{r \in(0, b) ; t<t_{s}\right\}$, and only the characteristic numbered 1 emanates from the "corner" $\left(b, t_{s}\right)$ into the region $\left\{r \in(0, b) ; t<t_{s}\right\}$. Therefore [5; p. 472] in a properly posed Cauchy problem, we must specify $g^{2}$ along the boundary $r=0$, and $g^{1}$ along the boundary $r=b$. This specification can be done by giving $g^{2}$ as a linear combination of the other $g^{k}$ plus a function of time along $r=0$ (and correspondingly for $g^{1}$ along $r=b$ ). But this is precisely the way we have stated our problem in section II, since

$$
\begin{array}{ll}
g^{2}=-g^{1} & \Leftrightarrow U=0 \\
g^{1}=g^{2}+c \quad\left(c=\left.\left(2 e^{\varphi / 2} \sqrt{-\frac{d p}{d v} v}\right)\right|_{v=v_{1}}\right) \Leftrightarrow v=v_{1} .
\end{array}
$$

Hence there exists a unique solution of our Cauchy problem in some neighbourhood to the past of the final surface.

Let $f(r, t)$ be this solution. If $t_{i}<t_{s}$, with $t_{s}-t_{i}$ sufficiently small, then the initial data given by $\left.f\right|_{t=t_{i}}(r)$ are regular, and evolve into a naked singularity.

By construction, our singularity has the property that the energy density approaches infinity as $\left(r_{s}, t_{s}\right)$ is approached. In Appendix II 
it it is shown that, in addition, there are incomplete causal geodesics terminating at the "sphere" $\left(r_{s}, t_{s}\right)$.

Finally, we consider the important question of stability.

First we extend the equation of state $p=p(v)$ to negative values of $v$, in any way that maintains the smoothness requirements on $p$. Having done this, there exists also a unique solution of our system of evolution equations to the future of the final surface. Since we have chosen the final data such that $\dot{v}\left(r_{s}, t_{s}\right)<0$, the specific volume $v$ takes on negative values to the future of the final surface. This formal extension therefore cannot be regarded as an extension of the spacetime; it is, however, useful for proving stability.

With the formal extension, our problem has a unique solution in a neighbourhood $\left\{t \in\left[t_{s}-h, t_{s}+h\right]\right\}$, for some $h>0$. In particular, if we choose our "initial" time $t_{i}$ such that $t_{s}-t_{i}<h$, then the initial data $\left.f\right|_{t=t_{i}}(r)$ evolve past $t_{s}$ into the region of negative $v$.

The formal extension shows that the occurrence of a shell crossing singularity is, in our formalism, a $C^{0}$ condition on the solution, and the nakedness conditions [Section III] are also $C^{0}$ conditions. Therefore, any solution of our system which lies in a sufficiently small uniform $C^{0}$ neighbourhood of the solution $f_{s}$ in the domain $\left\{t \in\left[t_{s}-h, t_{s}+h\right]\right\}$ has a naked singularity.

But it follows immediately from the convergence of the iteration procedure used in the existence proof for the Cauchy problem in Ref. [5; pp. 464-478] that given any uniform $C^{0}$ neighbourhood $N_{0}$ of the solution $f_{s}$, there exists a uniform $C^{2}$ neighbourhood $N_{1}$ of the initial data $f_{s t=t_{i}}(r)$ and a uniform $C^{3}$ neighbourhood $N_{2}$ of the equation of state $p=p(v)$ such that for any initial data in $N_{1}$ and any equation of state in $N_{2}$, the solution lies in $N_{0}{ }^{6}$.

That is to say, the occurrence of naked singularities treated in this paper is stable with respect to (spherically symmetric) initial data and equation of state.

\section{Conclusions and Remarks}

We have proved the following

Theorem. For any equation of state $p(v)$ which is $C^{3}$ for $v \in\left[0, v_{1}\right]$, with $d p / d v$ strictly negative $e^{7}$, there exist regular spherically symmetric initial data for Einstein's field equations such that

${ }^{6}$ The width $h>0$ of the strip in which the solution exists can be chosen uniformly for sufficiently small neighbourhoods $N_{1}, N_{2}$. Hence $t_{i}$ can be chosen such that every solution with initial data in $N_{1}$ and equation of state in $N_{2}$ actually can be extended by means of the iteration procedure beyond $t_{s}$ into the region of negative $v$.

7 This is equivalent to the demand that $\varepsilon^{2} d p / d \varepsilon$ is strictly positive. By the way, it is possible to weaken this condition to admit a large family of equations of state for which $d p / d v$ is allowed to be zero at $v=0$, but the proof is too tortuous to be worth explication. 
the unique maximally extended solution of Einstein's field equations with these initial data has a naked singularity,

moreover, this property is stable for arbitrary spherically symmetric perturbations of the initial data lying in a sufficiently small uniform $C^{2}$ neighbourhood, and arbitrary perturbations of the equation of state lying in a sufficiently small uniform $C^{3}$ neighbourhood.

Since our system of equations explicitely assumes spherical symmetry, the proof of stability based on those equations naturally holds only for spherically symmetric perturbations of the initial data. But because we need only $C^{0}$ conditions on the solutions for stability, it seems likely that one has stability also for nonspherical perturbations.

One is somewhat inclined to regard an equation of state in which the pressure is bounded as unphysical. We remind the reader that our upper bound on the pressure can be arbitrarily large: It is well to bear in mind that no assertions about the behaviour of the equation of state for pressures greater than, say, $10^{114} \mathrm{erg} / \mathrm{cm}^{3}$ can be taken very seriously, since in this regime our whole present theoretical framework can hardly be considered meanigful. Indeed, the most sensible approach to this matter is probably to accept the suggestion of Hawking and Ellis $\left[6 ;\right.$ p. 363] that any region with radius of curvature of the order $10^{-15} \mathrm{~cm}$ "for all practical purposes could be regarded as a singularity".

The results reported in this paper make it plausible that naked "singularities" of this much weaker sort can occur for any smooth monotonic equation of state $p=p_{1}(\varepsilon)$, since, given $p_{1}$, one can find another equation of state $p=p_{2}(\varepsilon)$ such that $p_{2}$ satisfies the restrictions of the above theorem and $p_{1}=p_{2}$ for $\varepsilon \leqq 10^{114} \mathrm{erg} / \mathrm{cm}^{3}$, say. Unfortunately, our theorem is only a local existence theorem; therefore it does not amount to a proof that the development into the past can be extended to reach "reasonable" initial data. Here "reasonable" would mean roughly a state in which, throughout the interior region, $\varepsilon\left(v_{1}\right) \leqq \varepsilon<\varepsilon_{2}$, where $\varepsilon_{2}$ is, say, of the order of $10^{10} \mathrm{~g} / \mathrm{cm}^{3}$, and the distribution of density and velocity is not too strange.

Returning again to the more stringent definition of singularity, we remark that there appears to be some small hope of extending our present method to the case of equations of state for which the pressure is a logarithmic function of the energy density, and to singularities which occur at the center of the object.

Acknowledgement. We thank Prof. J. Ehlers and Prof. A. H. Taub for helpful critical comments.

\section{Appendix I}

The existence and uniqueness proof for hyperbolic first order systems in Ref. [5] uses the smoothness requirements of Section IV in order to get estimates on certain integrals (for instance, see the top of [5; p. 468]). 
These estimates are needed to prove that the iteration operator is contracting. If the smoothness conditions hold, one can apply the mean value theorem directly to obtain the required estimates, as done in [5].

In our case, the smoothness conditions are violated at $r=0$, but in such a way that the required estimates can be obtained by a different method than that used in [5], so that the existence and uniqueness theorem still holds. Namely, a number of our coefficients are proportional to $R^{2} / r^{2}$, so that the first derivatives of these coefficients with respect to $R$ and $r$ are unbounded at $r=0$ (since $R$ is proportional to $r$ in a neighbourhood of $r=0$ ). The violation of the smoothness conditions is always of this particular type. Let us then see how we can get estimates on integrals of the general form

$$
\begin{aligned}
\mathscr{I} & :=\int_{0}^{h}\left[\left(\frac{R_{1}}{r}\right)^{2} F_{1}-\left(\frac{R_{2}}{r}\right)^{2} F_{2}\right] d t \\
& =\int_{0}^{h}\left[\left(\frac{R_{1}}{r}\right)^{2}\left(F_{1}-F_{2}\right)+\left(\left(\frac{R_{1}}{r}\right)^{2}-\left(\frac{R_{2}}{r}\right)^{2}\right) F_{2}\right] d t
\end{aligned}
$$

where $F_{n}:=F\left(r, t, f_{n}\right)$ has Lipschitz continuous first derivatives.

From the first constraint Eq. (6),

so that

$$
R^{3}=\int_{0}^{r} 3 r^{2} \Gamma v d r
$$

$$
\left\|\left(\frac{R}{r}\right)^{2}\right\| \leqq\|\Gamma v\|^{2 / 3} .
$$

Thus

$$
\mathscr{I} \leqq h\left\|\Gamma_{1} v_{1}\right\|^{2 / 3}\left\|F_{1}-F_{2}\right\|+\int_{0}^{h}\left\|\left[\left(\frac{R_{1}}{r}\right)^{2}-\left(\frac{R_{2}}{r}\right)^{2}\right] F_{2}\right\| d t .
$$

For the second term, note that the mean value theorem implies

$$
\left(\frac{R_{1}}{r}\right)^{2}-\left(\frac{R_{2}}{r}\right)^{2}=K(r)\left[\left(\frac{R_{1}}{r}\right)^{3}-\left(\frac{R_{2}}{r}\right)^{3}\right],
$$

with $K(r)$ finite for all $r$. Therefore,

$$
\begin{aligned}
& \left\|\left(\frac{R_{1}}{r}\right)^{2}-\left(\frac{R_{2}}{r}\right)^{2}\right\| \leqq\|K\|\left\|\frac{1}{r^{3}} \int_{0}^{r} 3 r^{2}\left(\Gamma_{1} v_{1}-\Gamma_{2} v_{2}\right) d r\right\| \\
& \leqq\|K\|\left\|\frac{1}{r^{3}}\right\| \Gamma_{1} v_{1}-\Gamma_{2} v_{2}\left\|\int_{0}^{r} 3 r^{2} d r\right\|=\|K\|\left\|\Gamma_{1} v_{1}-\Gamma_{2} v_{2}\right\| .
\end{aligned}
$$

Thus

$$
\mathscr{I} \leqq h\left\|\Gamma_{1} v_{1}\right\|^{2 / 3}\left\|F_{1}-F_{2}\right\|+h\left\|F_{2}\right\|\|K\|\left\|\Gamma_{1} v_{1}-\Gamma_{2} v_{2}\right\|,
$$

which can now be dealt with by routine methods. 


\title{
Appendix II
}

We show here that there exists an incomplete timelike geodesic which terminates on the singular "sphere" $\left(r_{s}, t_{s}\right)$.

Let $\left\{t_{n}\right\}$ be a monotonically increasing sequence which approaches $t_{s}$, and let $P_{n}$ be the point with coordinates $\left(r_{s}, \pi / 2,0, t_{n}\right)$, for each $n$. Define $K_{n}=J^{+}\left(P_{0}\right) \cap J^{-}\left(P_{n}\right)$; this is compact for each $n$, whence there exist timelike geodesics $g_{n}$ between $P_{0}$ and $P_{n}$ for each $n$. Some subsequence of the $g_{n}$ tends to a causal geodesic $g$ in some neighbourhood of $P_{0}$. In fact, $g$ cannot be null, for $t$ is a global time function in (the regular part of) our spacetime, which implies that the points of intersection of the past light cones of the $P_{n}$ with the future light cone of $P_{0}$ tend to limit spheres with $t<t_{s}$.

Finally, we note that $\frac{d s}{d t} \leqq e^{\varphi}$ along any timelike curve. Since $e^{\varphi}$ is bounded, $g$ has finite proper length, and is therefore an incomplete timelike geodesic terminating on the singular "sphere" $\left(r_{s}, t_{s}\right)$.

\section{References}

1. Yodzis, P., Seifert,H.J., Müller zum Hagen,H.: Commun. math. Phys. 34, 135-148 (1973)

2. Carter, B.: Phys. Rev. Letters 26, 331-333 (1971)

3. Misner, C. W., Sharp,D. H.: Phys. Rev. 136, B571-576 (1964)

4. Misner, C.W.: Gravitational collapse. In: Chrétien, M., Deser,S., Goldstein,J. (Eds.): Astrophysics and general relativity, pp. 113-216. New York: Gordon and Breach 1969

5. Courant, R., Hilbert, D.: Methods of mathematical physics; Volume II, Partial differential equations, third printing. New York-London-Sydney: Interscience Publishers 1966

6. Hawking, S.W., Ellis, G.F.R.: The large scale structure of space-time. Cambridge: At the University Press 1973

Communicated by J. Ehlers

\author{
H. Müller zum Hagen \\ P. Yodzis \\ I. Institut für Theoretische Physik \\ Universität Hamburg \\ D-2000 Hamburg 36 \\ Jungiusstraße 9 \\ Federal Republic of Germany \\ H.-J. Seifert \\ Hochschule der Bundeswehr Hamburg \\ Fachbereich Maschinenbau \\ D-2000 Hamburg 70 \\ Rodigallee 100 \\ Federal Republic of Germany
}

